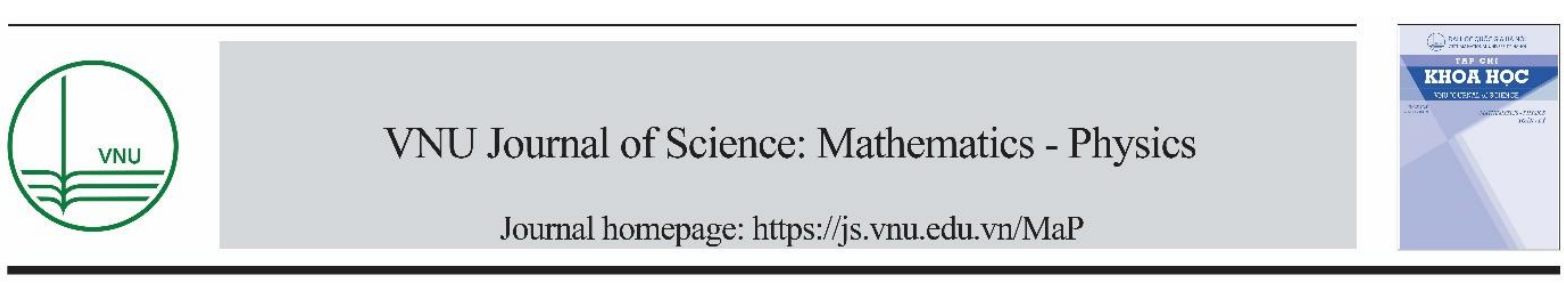

Original Article

\title{
The Calculation of the Ettingshausen Coefficient in Quantum Wells under the Influence of Confined Phonons (for Electron - confined Optical Phonon Scattering)
}

\author{
Nguyen Thi Lam Quynh", Cao Thi Vi Ba, Nguyen Quang Bau \\ Faculty of Physics, VNU University of Science, \\ 334 Nguyen Trai, Hanoi, Vietnam
}

Received 22 March 2019

Accepted 14 May 2019

\begin{abstract}
In this paper, we have used the method of quantum kinetic equation to calculate the analytic expression for Ettingshausen coefficient (EC) under the influence of confined phonon. We considered a quantum well in the presence of constant electric field, magnetic field and electromagnetic wave (EMW) with assumption that electron - confined optical phonon (OP) scattering is essential. The EC obtained depends on many quantities in a complicated way such as temperature, magnetic field, frequency or amplitude of EMW and $m$ - quantum number which specify confined OP. Numerical results for GaAs/GaAsAl quantum well (QW) have displayed clearly the differences in comparison with both cases of bulk semiconductor and unconfined phonon. The result of examining the EC's dependence on magnetic field shows that quantum number $m$ changes resonance condition; $m$ not only makes the increase in the number of resonance peak but also changes the position of peaks. When $m$ is set to zero, we get the results that corresponds to unconfined OP.
\end{abstract}

Keywords: Quantum well, Ettingshausen effect, Quantum kinetic equation, confined optical phonons.

\section{Introduction}

Due to the confinement effect, the movement of electron and phonon is severely limited. This leads to changes in characteristics of quantum effects appeared in low-dimensional semiconductor systems (LDS), in particular two-dimensional systems [1-5].

\footnotetext{
${ }^{*}$ Corresponding author.

Email address: lamquynh.katty@gmail.com
}

https//doi.org/ 10.25073/2588-1124/vnumap.4338 
In comparison with the case of unconfined phonons, many published works indicated that the confinement of phonons creates new behaviours of materials under external stimulies [1-3]: the confinement of LO-phonons makes a remarkable impact on the magneto-phonon resonance condition in doped semiconductor supperlatices [1]; in compositional supperlatices, confined optical phonons make the nonlinear absorption of a strong electromagnetic wave by confined electrons [2] and increase the number of resonance peaks of the Hall conductivity [3].

The Ettingshausen effect has been studied in semiconductor [6] and quantum wells [4, 5]. In [4, 5], properties of this magneto-thermoelectric effect are different from bulk semiconductor [6] due to the confinement of the electrons. However, the confinement of phonons, in particular OP, has not been interested yet. In this work, we study the influence of confined OP on the EC in QW. The report is structured as follows: in section 2, we report the impact of confined OP on the EC in QW; section 3 gives the numerial results and discussion for GaAs/GaAsAl QW; conclusions are shown in section 4 .

\section{The Ettingshausen coefficient in quantum wells under the influence of confined optical phonons}

To obtain the analytic expression for the EC, we proceed in turn: (1) establish the quantum kinetic equation for electron distribution function by using the Hamiltonian of electron - confined optical phonon system; (2) solve the quantum kinetic equation obtained to get the total current density and the thermal flux density; (3) calculate the tensor and deduce the expression for the EC.

Model of QW used is put into a parabolic potential with confined frequency $\omega_{z}$ [4]. So, motion of the electrons is free in the $\mathrm{x}-\mathrm{y}$ plane. QW is also considered in the presence of constant electric field $\overrightarrow{E_{1}}=\left(E_{1}, 0,0\right)$, magnetic field $\vec{B}=(0,0, B)$ (the magnetic field is perpendicular to the free-moving plane of electrons) and EMW $\vec{E}=E_{0} \sin (\omega t)$. From the Hamiltonian of electron-confined OP in QW, quantum kinetic equation for electrons is obtained by using the commutative relations of creation and annihilation operators. After some analytic tranformations, we found out the expression for tensors:

$$
\begin{aligned}
& \sigma_{i p}(m)=a \frac{e \tau\left(\varepsilon_{F}\right)}{1+\omega_{c}^{2} \tau^{2}\left(\varepsilon_{F}\right)} \delta_{k p} \delta_{01}+\frac{e}{m_{e}}\left\{a_{1}(m) \frac{\tau_{1}^{2}(\varepsilon)}{\left[1+\omega_{c}^{2} \tau_{1}^{2}(\varepsilon)\right]^{2}} \delta_{1}+a_{2}(m) \frac{\tau_{2}^{2}(\varepsilon)}{\left[1+\omega_{c}^{2} \tau_{2}^{2}(\varepsilon)\right]^{2}} \delta_{2}\right. \\
& +a_{3}(m) \frac{\tau_{3}^{2}(\varepsilon)}{\left[1+\omega_{c}{ }^{2} \tau_{3}^{2}(\varepsilon)\right]^{2}} \delta_{3}+a_{4}(m) \frac{\tau_{4}^{2}(\varepsilon)}{\left[1+\omega_{c}{ }^{2} \tau_{4}^{2}(\varepsilon)\right]^{2}} \delta_{4}+a_{5}(m) \frac{\tau_{5}^{2}(\varepsilon)}{\left[1+\omega_{c}^{2} \tau_{5}^{2}(\varepsilon)\right]^{2}} \delta_{5} \\
& \left.+a_{6}(m) \frac{\tau_{6}^{2}(\varepsilon)}{\left[1+\omega_{c}^{2} \tau_{6}^{2}(\varepsilon)\right]^{2}} \delta_{6}+a_{7}(m) \frac{\tau_{7}^{2}(\varepsilon)}{\left[1+\omega_{c}^{2} \tau_{7}^{2}(\varepsilon)\right]^{2}} \delta_{7}+a_{8}(m) \frac{\tau_{8}^{2}(\varepsilon)}{\left[1+\omega_{c}{ }^{2} \tau_{8}^{2}(\varepsilon)\right]^{2}} \delta_{8}\right\} \\
& \varphi_{i p}(m)=-\frac{1}{m_{e} T}\left[a_{1}(m) b(m)_{1} \delta_{1}+a_{2}(m) b_{2}(m) \delta_{2}+a_{3}(m) b_{3}(m) \delta_{3}\right. \\
& \left.+a_{4}(m) b_{4}(m) \delta_{4}+a_{5}(m) b_{5}(m) \delta_{5}+a_{6}(m) b_{6}(m) \delta_{6}+a_{7}(m) b_{7}(m) \delta_{7}+a_{8}(m) b_{8}(m) \delta_{8}\right] \\
& \eta_{i p}(m)=-\frac{1}{m_{e}}\left[a_{1}(m) b(m)_{1} \delta_{1}+a_{2}(m) b_{2}(m) \delta_{2}+a_{3}(m) b_{3}(m) \delta_{3}\right. \\
& \left.+a_{4}(m) b_{4}(m) \delta_{4}+a_{5}(m) b_{5}(m) \delta_{5}+a_{6}(m) b_{6}(m) \delta_{6}+a_{7}(m) b_{7}(m) \delta_{7}+a_{8}(m) b_{8}(m) \delta_{8}\right]
\end{aligned}
$$




$$
\begin{aligned}
& \mu_{i p}(m)=-\frac{1}{e m_{e} T}\left\{a_{1}(m) b_{1}^{2}(m) \frac{\tau_{1}^{2}(\varepsilon)}{\left[1+\omega_{c}{ }^{2} \tau_{1}^{2}(\varepsilon)\right]^{2}} \delta_{1}+a_{2}(m) b_{2}^{2}(m) \frac{\tau_{2}{ }^{2}(\varepsilon)}{\left[1+\omega_{c}{ }^{2} \tau_{2}{ }^{2}(\varepsilon)\right]^{2}} \delta_{2}\right. \\
& +a_{3}(m) b_{3}^{2}(m) \frac{\tau_{3}^{2}(\varepsilon)}{\left[1+\omega_{c}{ }^{2} \tau_{3}^{2}(\varepsilon)\right]^{2}} \delta_{3}+a_{4}(m) b_{4}{ }^{2}(m) \frac{\tau_{4}{ }^{2}(\varepsilon)}{\left[1+\omega_{c}{ }^{2} \tau_{4}{ }^{2}(\varepsilon)\right]^{2}} \delta_{4} \\
& +a_{5}(m) b_{5}{ }^{2}(m) \frac{\tau_{5}{ }^{2}(\varepsilon)}{\left[1+\omega_{c}{ }^{2} \tau_{5}^{2}(\varepsilon)\right]^{2}} \delta_{5}+a_{6}(m) b_{6}{ }^{2}(m) \frac{\tau_{6}{ }^{2}(\varepsilon)}{\left[1+\omega_{c}{ }^{2} \tau_{6}{ }^{2}(\varepsilon)\right]^{2}} \delta_{6} \\
& \left.+a_{7}(m) b_{7}{ }^{2}(m) \frac{\tau_{7}{ }^{2}(\varepsilon)}{\left[1+\omega_{c}{ }^{2} \tau_{7}{ }^{2}(\varepsilon)\right]^{2}} \delta_{7}+a_{8}(m) b_{8}{ }^{2}(m) \frac{\tau_{8}{ }^{2}(\varepsilon)}{\left[1+\omega_{c}{ }^{2} \tau_{8}{ }^{2}(\varepsilon)\right]^{2}} \delta_{8}\right\}
\end{aligned}
$$

Where: $\omega_{c}=\frac{e B}{m_{e}}$ is cyclotron frequency of electron; $\tau_{s}(\varepsilon)$ is the momentum relaxation time.

$$
a=-\frac{e \beta \hbar v_{d} n_{0}}{m_{e}} I\left(k_{y}\right) \sum_{N, n} e^{\beta\left(\varepsilon_{F}-\varepsilon_{N, n}\right)}
$$

$$
b_{s}(m)=B_{1}-e E_{1} \bar{l} \pm \hbar \omega_{m}(s=1 \div 4) ; b_{s}(m)=B_{1}-e E_{1} \bar{l} \pm \hbar \omega_{m} \pm \hbar \omega(s=5 \div 8)
$$

$a_{s}(m)=A \sum_{N, n^{\prime}, m} \sum_{N, n} I_{n, n^{\prime}}^{m}\left(\frac{e E_{1}}{\hbar v_{d}} \bar{l}\right) e^{\beta\left(\varepsilon_{F}-\varepsilon_{N, n}\right)} I\left(k_{y}\right) f(N) \delta\left(B_{1}-e E_{1} \bar{l} \pm \hbar \omega_{m}\right)(s=1,2)$

$a_{s}(m)=-\frac{\kappa}{2} A \sum_{N^{\prime}, n^{\prime}, m} \sum_{N, n} I_{n, n^{\prime}}^{m}\left(\frac{e E_{1}}{\hbar v_{d}}\right)^{3} e^{\beta\left(\varepsilon_{F}-\varepsilon_{N, n}\right)} I\left(k_{y}\right) f(N) \delta\left(B_{1}-e E_{1} \bar{l} \pm \hbar \omega_{m}\right)(s=3,4)$

$a_{s}(m)=\frac{\kappa}{4} A \sum_{N, n^{\prime}, m} \sum_{N, n} I_{n, n^{\prime}}^{m}\left(\frac{e E_{1}}{\hbar v_{d}} \bar{l}\right)^{3} e^{\beta\left(\varepsilon_{F}-\varepsilon_{N, n}\right)} I\left(k_{y}\right) f(N) \delta\left(B_{1}-e E_{1} \bar{l} \pm \hbar \omega_{m} \pm \hbar \omega\right)(s=5 \div 8)$

with defination: $\bar{l}=\left(\sqrt{N+\frac{1}{2}}+\sqrt{N+1+\frac{1}{2}}\right) \frac{l_{B}}{2}[1] ; \varepsilon_{F}$ is the Fermi level.

$$
\begin{gathered}
\kappa=\left(\frac{e E_{0}}{m_{e} \omega}\right)^{2} ; \omega_{m}=\sqrt{\omega_{o}^{2}-v_{s}^{2}(m \pi / L)^{2}} ; I_{n, n^{\prime}}^{m}=\left|\frac{1}{L \sqrt{\pi 2^{n} n ! 2^{n^{\prime}} n ! !}} \int_{-\infty}^{+\infty} e^{i \frac{m \pi}{L} z} e^{-\frac{z^{2}}{L^{2}}} H_{n}\left(\frac{z}{L}\right) H_{n^{\prime}}\left(\frac{z}{L}\right) d z\right|^{2} \\
I\left(k_{y}\right)=\frac{L_{y}}{2 \pi}\left[\frac{1}{\beta \hbar v_{d}}\left(e^{\beta \hbar v_{d} \frac{L_{x}}{2 l}}+e^{-\beta \hbar v_{d} \frac{L_{x}}{2 l}}\right)-\frac{1}{\left(\beta \hbar v_{d}\right)^{2}}\left(e^{\beta \hbar v_{d} \frac{L_{x}}{2 l}}-e^{-\beta \hbar v_{d} \frac{L_{x}}{2 l}}\right)\right] \\
A=\frac{4 \pi^{2} e^{3} \hbar \omega_{m} n_{0} \beta}{\varepsilon_{0} m_{e}} \frac{1}{e^{\beta h \omega_{m}}-1}\left(\frac{1}{\chi_{\infty}}-\frac{1}{\chi_{0}}\right) ; B_{1}=\left(N^{\prime}-N\right) \hbar \omega_{c}+\left(n^{\prime}-n\right) \hbar \omega_{z} \\
\varepsilon_{N, n}=\left(N+\frac{1}{2}\right) \hbar \omega_{c}+\left(n+\frac{1}{2}\right) \hbar \omega_{z}+\frac{1}{2} m_{e} v_{d}{ }^{2} ; f(N)=\frac{1}{4 \pi\left(N_{2}-N_{1}\right)}\left[1-\frac{l^{2}(m \pi)^{2}}{2 L^{2}\left(N_{2}-N_{1}-1\right)}\right] \\
\delta_{n}=\left[\delta_{i k}+\omega_{c} \tau(\varepsilon) \epsilon_{i j k} h_{j}-\omega_{c}{ }^{2} \tau^{2}(\varepsilon) h_{i} k_{k}\right]\left[\delta_{k p}+\omega_{c} \tau(\varepsilon) \in_{k l p} h_{l}-\omega_{c} \tau^{2}(\varepsilon) h_{k} k_{p}\right](n=1 \div 7)
\end{gathered}
$$


$\delta_{i k}$ is the Kronecker delta; the Latin symbols $i, j, k, l, p$ stand for the components $\mathrm{x}, \mathrm{y}, \mathrm{z}$ of the Cartesian coordinates; $\epsilon_{k l p}$ is the anti-symmetrical Levi - Civita tensor.

From analytic expression of tensors given by Eq.1, Eq.2, Eq.3 and Eq.4, we can calculate their components, those are $\sigma_{x x}(m), \sigma_{x y}(m), \varphi_{x x}(m), \eta_{x x}(m), \eta_{x y}(m), \mu_{x x}(m)$, respectively. The EC is written by:

$$
E c=\frac{1}{H} \frac{\sigma_{x x}(m) \eta_{x y}(m)-\sigma_{x y}(m) \eta_{x x}(m)}{\sigma_{x x}\left\{\varphi_{x x}(m) \eta_{x x}(m)-\sigma_{x x}(m)\left[\mu_{x x}(m)-K_{L}\right]\right\}}
$$

Here: $K_{L}$ is the thermal conductivity of phonons.

According to Eq.5, the EC depends on many quantities such as temperature, ampitude and frequency of EMW, magnetic field. Especially, the EC depends in a complicated way on the $m$-quantum number specific confinement of phonons. It means the change of OP for wave vector and frequency due to confinement has significant effect on theoretical results. In particular, the results for the case of unconfined OP are obtianed when we set $m$ to zero.

\section{Numerical results and discussions}

We have considered a QWPP of GaAs/GaAsAl subjected to uniform crossed magnetic field, constant electric field in the presence of an EMW to get the influence of the confined optical phonon on the EC. Parameters used are as follows: $v_{s}=87300 \mathrm{~ms}^{-1}[2,3], N=1, N^{\prime}=3$ [3], $\mathrm{n}$ and n' rate from 1 to $3, m_{e}=0.067 m_{0}, e=2.07 e_{0} \chi_{0}=12.9, \chi_{\infty}=10.9, \varepsilon_{F}=50 \mathrm{meV}[5], L_{x}=L_{y}=2 \mathrm{~nm}, \omega_{z}=10^{12} \mathrm{~Hz}$.

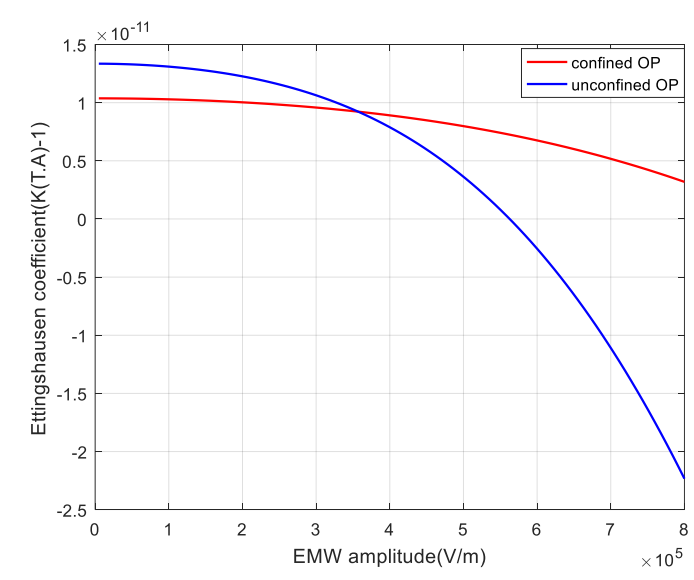

Figure 1. The dependence of EC on EMW amplitude with $\omega=5.10^{13} \mathrm{~Hz}, T=300 \mathrm{~K}, B=1 T$

Fig.1 indicates the influence of confined OP on the EC. In investigated range, the EC decreases as the EMW increases in both cases (with and without the confinement of OP). The EC increases fast when EMW amplitude is greater than $2.10^{5} \mathrm{~Hz}$ and got negative values in higher amplitude domain ( $\geq 6.10^{5} \mathrm{~Hz}$ ) in the case of unconfined OP. Meanwhile, due to the confinement phonon, the EC decreases more slowly and only has positive values in the whole survey amplitude region. 


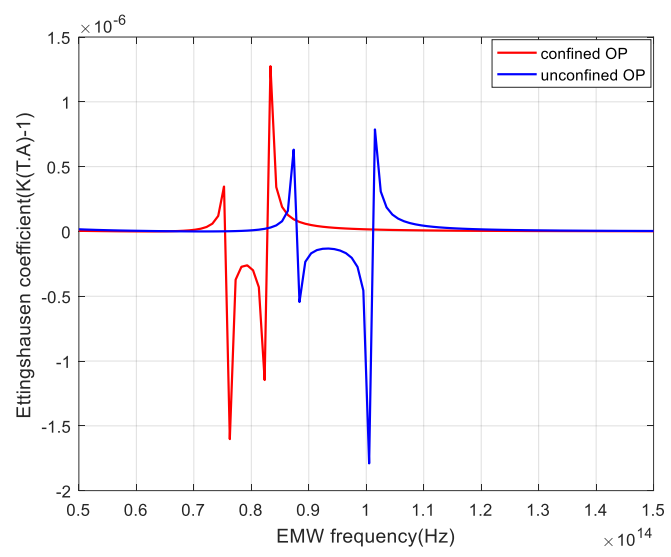

Figure 2. The dependence of EC on EMW frequency with $\omega=5.10^{13} \mathrm{~Hz}, T=300 \mathrm{~K}, B=1 T$

Fig.2 shows that in this EMW frequency range, peaks of the red line (with confined OP) are sideways to the left in comparison with peaks of the blue line (with unconfined OP). It can be explained easily as follows: resonance peaks correspond to the condition: $2 \omega_{c}+\left(n^{\prime}-n\right) \omega_{z} \pm \omega_{m} \pm \omega=0$ with $\omega_{m}=\sqrt{\omega_{0}^{2}-v_{s}^{2}(m \pi / L)^{2}}$; thus, when OP are confined $(m \geq 1), \omega_{m}$ decreases and the resonance peaks move to another frequency area.

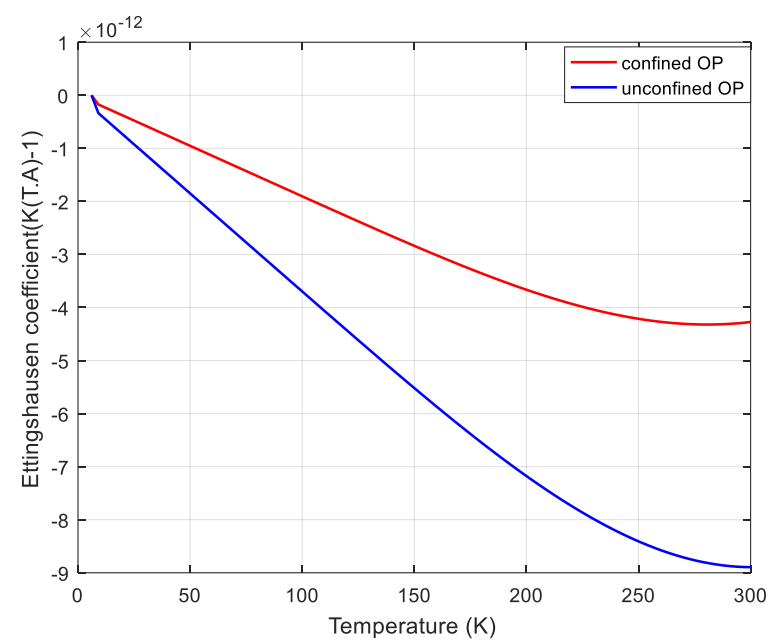

Figure 3. The dependence of EC on temperature with $B=0.1 T, \omega=5.10^{13} \mathrm{~Hz}$

As we see in Fig.3, the EC depends on temperature in a non-linear way. According to graph, the EC decreases fast and nearly linear when the temperature increases from $0 \mathrm{~K}$ to $250 \mathrm{~K}$; decreasing speed of the EC is slower when the temperature is between $250 \mathrm{~K}$ and $300 \mathrm{~K}$. The graph displayed clearly that the EC has negative value in both case with and without confinement of OP. This is contrary to published results of the EC in the same QW with unconfined OP [4]. The cause of this difference is the direction of the magnetic field. In the previous study, the EC was investigated in terms of the magnetic field lying in the plane where the electrons moved freely and had positive values. Meanwhile, we study the EC when the magnetic field is perpendicular to the free-moving plane of electrons. 


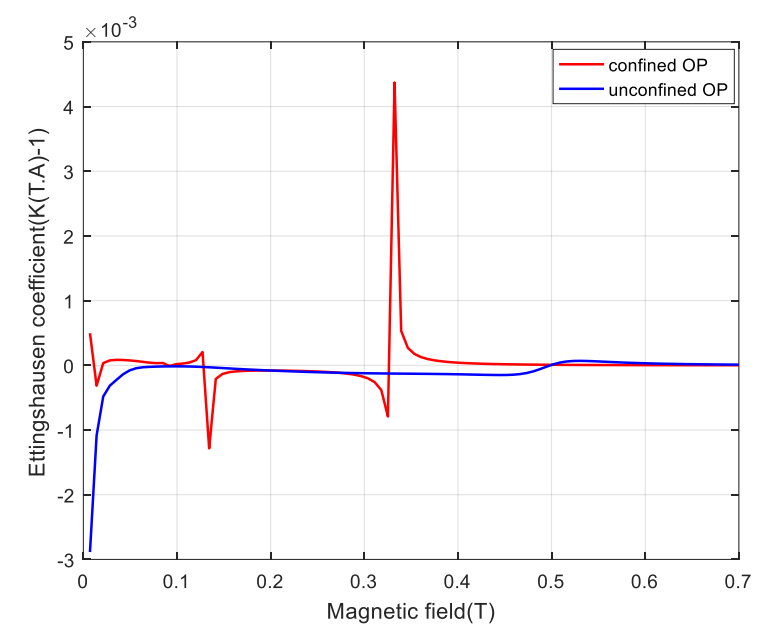

Figure 4. The dependence of EC on magnetic field with $T=300 \mathrm{~K}, \omega=2,6.10^{13} \mathrm{~Hz}$

According to Fig.4, the EC oscillates as the magnetic field increases whether OP is confined or not. Due to the OP confinement, the number of resonance peaks enhance. Furthermore, the peak positions of the red line are shifted to the left in comparison with the blue line. These peaks correspond to the condition: $2 \omega_{c}=\left(n-n^{\prime}\right) \omega_{z}+e E_{1} \bar{l} \pm \omega_{m} \pm \omega$. When OP are confined, thier frequency is defined: $\omega_{m}=\sqrt{\omega_{0}^{2}-v_{s}^{2}(m \pi / L)^{2}}$. So, $\omega_{m}$ is affected by the increase of quantum number $m$. The larger value of $m$, the more resonance peaks appear.

\section{Conclusions}

By using quantum kinetic equation method, we have found out the analytic expressions for the conductivity tensors and the $\mathrm{EC}$ in $\mathrm{QW}$ of GaAs/GaAsAl under the influence of confined OP. Due to significant contribution of the confined OP, theoretical results are different from the previous researches for Ettingshausen effect in QW $[4,5]$. The increase of confinement effect of OP impacts the resonant condition in QW under the influence of external field and leads to the decrease of the EC. The more confinement effect of OP, the more resonance peaks of the EC appear. In other hand, when temperature or EMW amplitude increases, the EC decreases. When we set quantum number $m$ specific the OP confinement to zero, the results we get are fit to the case of unconfined OP. So far, the results obtained contribute to the theory of quantum effect in LDS.

\section{Acknowledgments}

This work was completed with financial support from the National Foundation for Science and Technology Development of Vietnam (Nafosted 103.01-2015.22). 


\section{References}

[1] N.Q. Bau, D.T. Long, Impact of confined LO-phonons on the Hall effect in doped semiconductor supperlatices, Journal of Science: Advanced Materials and Devices 1 (2016) 209-213.

[2] L.T. Hung, N.V. Nhan, N.Q. Bau, The impact of confined phonons on the nolinear obsorption coefficient of a strong electromagnetic wave by confined electrons in compositional supperlatices, VNU Journal of Science, Mathematics - Physics 28 (2012) 68-76.

[3] N.Q. Bau, D.T. Long, Influence of confined optical phonons and laser radiation on the Hall effect in $a$ compositional supperlatices, Physica B:Condensed Matter 532 (2018) 149-154.

[4] N.Q. Bau, D.T. Hang, D.M. Quang, N.T.T. Nhan, Magneto-thermoelectric effect in quantum well in the presence of electromagnetic wave, VNU Journal of Science, Mathematics - Physics 32 (2017) 1-9.

[5] D.T. Hang, D.T. Ha, D.T.T. Thanh, N.Q. Bau, The Ettingshausen coefficient in quantum wells under the influence of laser radiation in the case of electron-optical phonon interaction, Photonics Letters of Poland, 8 (3) (2016) 79-81.

[6] B.V. Paranjape, J.S. Levinger, Theory of the Ettingshausen effect in emiconductors, Phys. Rev. 120 (1960) 437 441. 